\title{
Ventilator Boot Camp Improves the Knowledge and Skills Associated With Mechanical Ventilator Use During Interfacility Transport of Intubated Pediatric Patients
}

\author{
Jennifer L Walton RRT CNP-T EMT-B, Diane K Dunn RRT RRT-NPS, \\ Nhi Y Haines RRT RRT-NPS, Ilana Heisler MSc RRT, Michael T Bigham MD, and \\ Teresa A Volsko MBA MHHS RRT CMTE FAARC
}

\begin{abstract}
BACKGROUND: The American Academy of Pediatrics Section on Transport recommends the use of portable ventilators during the transport of patients with advanced airways. We sought to identify knowledge gaps and evaluate the effectiveness of a transport ventilator competency boot camp. METHODS: Electronic health records of children requiring ventilatory support during air and ground interfacility transport from January 1 through December 31, 2015, were reviewed to determine when manual ventilation was used in lieu of a portable ventilator, and simulations were constructed from commonly occurring scenarios. All registered respiratory therapists trained in air and ground critical-care transports participated. Demographic data were collected. We assessed performance on 3 facilitated simulated scenarios using a ventilator connected to a low-fidelity pediatric mannequin attached to breathing simulator. Scores were based on the participants' ability to correctly perform pre-use checks, select and optimize ventilator settings, set alarms, and complete safety checks. A 60-min interactive education intervention was conducted between the preand post-assessments. The pre-assessment, intervention, and post-assessment were conducted 6 weeks apart. De-identified assessments were scored, and results were shared after study completion. Descriptive statistics reported participant demographics. Paired $t$ tests compared before and after assessments. Statistical significance was established at $P<.05$. RESULTS: A total of 172 electronic health records were reviewed. Manual ventilation was used more frequently in toddlers requiring pressure control ventilation; noninvasive ventilation was rarely used. A total of 17 registered respiratory therapists participated; $18 \%$ were male, $41 \%$ had 6-9 years of tenure and 5 years of experience with our transport team. Completing ventilator pre-use check and engaging alarms provided the most opportunity for improvement. Improvements were greater with the use of noninvasive ventilation $(P=.006)$ than pressure control ventilation $(P=.10)$ and volume control ventilation use $(P=.07)$. CONCLUSIONS: Quality data were useful in identifying areas requiring knowledge and competency assessment. Re-assessment results validated the need to conduct education and competency assessment at defined intervals. Key words: mechanical ventilation; pediatric; transport. [Respir Care 2018;63(4):417-423. (C) 2018 Daedalus Enterprises]
\end{abstract}

Introduction

Interfacility patient transport allows access to specialized care, including advanced diagnostic and therapeutic procedures that are often unavailable at the referring facility. However, patient transport by air or ground carries an element of risk. Transport teams function in an environment where resources in terms of equipment, staffing, and support are limited.
Providing safe, quality care during interfacility transport is essential. The American Academy of Pediatrics Section on Transport Medicine along with the Air Medical Physician Association recognized this need and established the Ground and Air Medical Quality in Transport (GAMUT) Quality Improvement Collaborative. The GAMUT Quality Improvement Collaborative provided a platform for air and ground medical transport programs to collaborate, learn, and improve the care provided to patients en route to 
definitive care. Included in the recommended metrics from this collaborative is the use of portable mechanical ventilators for the transport of patients with advanced airways. Compared to manual ventilation with a bag-valve or flowinflating resuscitator, mechanical ventilators provide more consistent minute ventilation, minimizing alterations in blood gas results. ${ }^{1,2}$ Among the several metrics related to ventilation and airway care, air and ground medical transport programs report mechanical ventilator use during transport. The data shared through this quality collaborative facilitates comparison between transport programs and against national benchmarks. ${ }^{3}$ The use of a mechanical ventilator during interfacility transport of pediatric patients requiring invasive or noninvasive ventilator at our program was below the national benchmark. ${ }^{4}$

To improve this quality metric, we sought to perform a series of rapid-cycle process improvements or plan-dostudy-act (PDSA) cycles to address the barriers to portable ventilator use with pediatric patients requiring ventilatory assistance during air and ground interfacility transport. The specific aim of this study was to evaluate the effectiveness of a transport ventilator boot camp in identifying knowledge gaps, and to evaluate the effectiveness of educational interventions designed to improve competency with the use of the LTV 1200 (Vyaire Medical, Mettawa, IL, USA) ventilator for the interfacility transport of pediatric patients requiring invasive and noninvasive ventilatory support.

\section{Methods}

\section{Pre-study Procedures}

According to guidelines for participation in the GAMUT database, the number of pediatric patients transported with advanced airways (denominator) and the number of those who were supported by mechanical ventilator (numerator) was tracked monthly. Data were tracked using a run chart, with 8 points depicting baseline performance. The national benchmark for ventilator use during the transport of a patient with an advanced airway was $95 \% .^{4}$

To identify the factors contributing to below-benchmark performance of this metric, we convened a focus group of respiratory therapist, nurse, and paramedic transport crew members to address the barriers to portable mechanical ventilator use during transport. Figure 1 provides the pro-

\footnotetext{
Ms Walton, Dunn, and Haines are affiliated with the Department of Respiratory Care, Akron Children's Hospital, Akron, Ohio. Ms Heisler is affiliated with the Rush University, Respiratory Care Program, Chicago, Illinois, and with Boston Children's Hospital, Boston, Massachusetts. Dr Bigham is affiliated with the Division of Critical Care Medicine, Akron Children's Hospital, Akron, Ohio. Ms Volsko is affiliated with the Nursing Administration, Akron Children's Hospital, Akron, Ohio.
}

\section{QUICK LOOK}

\section{Current knowledge}

National quality benchmarks, such as the use of a portable ventilator to support the needs of patients requiring ventilatory assistance during interfacility transport, provide clinicians targets for better practices. Rapidcycle process improvements offer a structure to engage staff in small tests of change to improve quality metrics, meet national benchmarks, and employ and sustain better practices.

\section{What this paper contributes to our knowledge}

A longitudinal assessment of a quality performance metric is helpful in identifying process and non-process factors, such as knowledge deficits that contribute to performance that falls below benchmarks or standards. The use of rapid-cycle process improvements addressed the process factors and identified the need for and evaluation of a ventilator boot camp. The use of a ventilator boot camp facilitated incremental and sustained improvement in the respiratory therapist's knowledge which positively impacted the quality measure, ventilator use on transport.

cess, material, and equipment issues the transport crew identified. A series of rapid cycle improvements were conducted to address issues with the ventilator battery, mounting the ventilator, and carrying the ventilator to and from the facility and the transport vehicle.

To address the concern the focus group identified regarding the difficulty using the portable ventilator with small patients, an electronic health record review was conducted. The electronic health records of all children requiring ventilatory support during air and ground interfacility transport to our pediatric intensive care unit from January 1 through December 31, 2015 ( $n=172$ subjects) were reviewed to determine when manual ventilation was used in lieu of a portable ventilator. Manual ventilation was used more frequently in toddlers requiring pressure control ventilation and when volume control ventilation was required for status epilepticus. In lieu of noninvasive ventilation (NIV), mask CPAP with a flow-inflating bag or bag-mask ventilation was used. We constructed simulated

Correspondence: Teresa A Volsko MBA MHHS RRT CMTE FAARC, Akron Children's Hospital, 1 Perkins Square, Akron, Ohio 44308. E-mail: tvolsko@akronchildrens.org.

DOI: $10.4187 /$ respcare.05951 


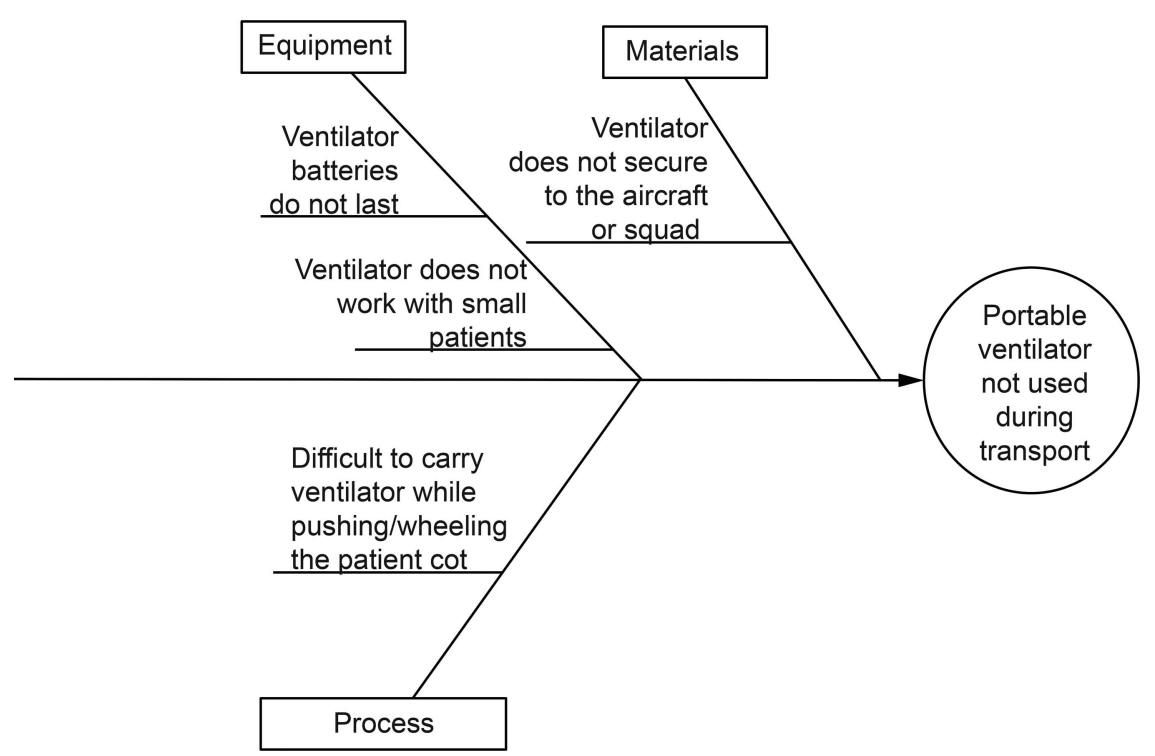

Fig. 1. A fishbone diagram depicting the process, equipment, and material factors impeding the use of a portable ventilator for pediatric transport.

scenarios for the ventilator boot camp from the most frequently occurring clinical situations where manual rather than mechanical ventilation was used during transport.

\section{Participant Enrollment and Study Procedures}

The study protocol was approved by the institutional review board, and a waiver of assent and informed consent was granted for this quality-improvement initiative. In addition, all registered respiratory therapists (RT) trained in air and ground critical-care transports participated in this quality initiative. There were 3 arms to the ventilator boot camp study: a pre-assessment, an educational intervention, and a post-assessment. The pre-assessment provided a baseline of the RTs' knowledge and skills. The educational intervention was an interactive session that reviewed the key concepts incorporated in the pre-and post-assessments. The post-assessment evaluated the knowledge and skills of the RTs after participation in the educational intervention.

Three scenarios were used in the pre-assessment and in the post-assessment phases: a 2-month-old male infant with respiratory distress requiring pressure control ventilation, a 10-y-old male with status epileptic requiring volume control ventilation, and a 16 -y-old female with severe $\operatorname{cog}$ nitive deficiency requiring noninvasive ventilation. Three testing stations were established, one for each of these scenarios. Each testing station had a low-fidelity pediatric mannequin that was connected to an ASL 5000 breathing simulator (IngMar Medical, Pittsburgh, PA, USA) to simulate active breathing. The breathing simulator was programmed to provide the breathing frequency and breathing pattern for each of the simulated scenarios. The RT attached the mannequin to the LTV 1200 ventilator by an endotracheal tube when the RT determined invasive ventilation was indicated, or by a mask when NIV was indicated. Vital signs, specifically heart rate and rhythm, breathing frequency, blood pressure, temperature, and $\mathrm{S}_{\mathrm{pO}_{2}}$ were displayed on a computer screen configured to display these vital signs in the same way they would be on a cardiorespiratory monitor. Vital signs were manually changed by the proctor, depending on the individual respiratory therapist's ability to manage the ventilator. For example, if the respiratory therapist selected the appropriate ventilator settings, improvements in vital signs, including $\mathrm{S}_{\mathrm{pO}_{2}}$, would be displayed on the computer screen. The proctor provided additional feedback on ausculatory findings, chest rise, and capillary refill when the RT inquired. Once the RT connected the end-tidal $\mathrm{CO}_{2}$ monitor in-line with the ventilator, the computer displayed end-tidal $\mathrm{CO}_{2}$. Participants were scored on their ability to correctly perform pre-use checks, select the mode of ventilation and initial ventilator settings, optimize ventilator settings to provide adequate ventilation within institutional guidelines for lung-protective strategies, set alarms appropriately, and complete safety checks.

The pre- and post-assessments were proctored. An envelope was provided to each participant. The participants were required to complete all 3 assessment stations. Only 1 participant was assessed at a station at a time. After the simulation, the proctor folded the completed assessment sheet to mask the assessment rating and placed it in an envelope. The envelope was then sealed and placed in the participant's master envelope. This process was repeated un- 


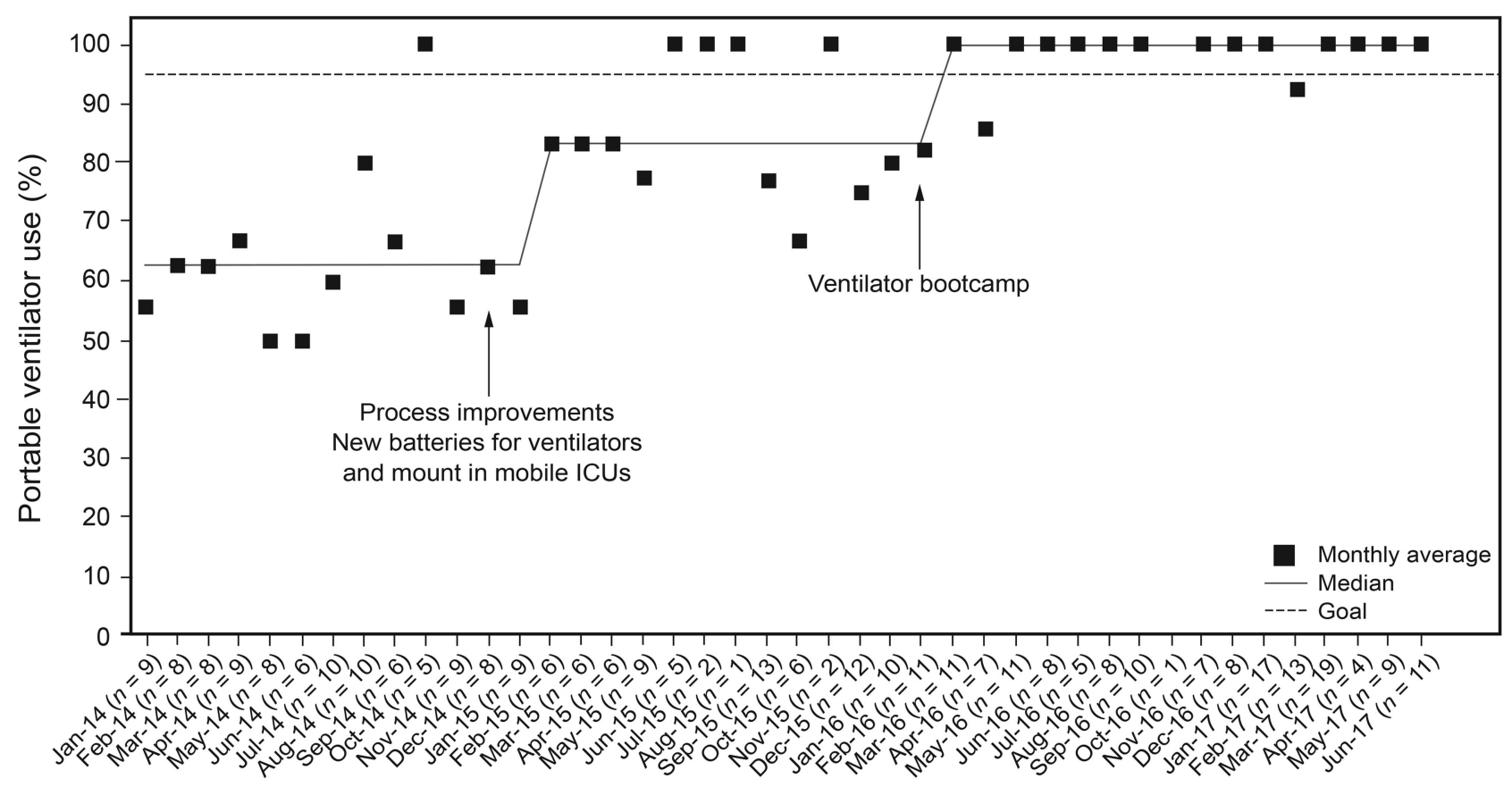

Month-Year

Fig. 2. A run chart depicting the cumulative effect of the plan-do-study-act cycles and the ventilator boot camp on portable ventilator use during interfacility transport.

til each participant completed all 3 assessment stations. Each master envelope was assigned a unique identifier after completion. The participant was not aware of the identifier to which they were assigned. Each participant also completed a brief survey to ascertain demographics, including gender, age, highest completed academic degree, professional longevity, and years of air and ground transport experience. The survey was anonymous and confidential.

The pre-assessment, educational intervention, and post-assessment phases were conducted 6 weeks apart. The participants were unaware of their scores during their participation in the study. Assessment scores were shared confidentially with each participant after study completion.

The intervention, a 60-min interactive education session, was conducted between the pre-assessment and the post-assessment. The intervention addressed all of the tasks associated with safety and pre-use checks, the use of lungprotective strategies when selecting tidal volume $\left(\mathrm{V}_{\mathrm{T}}\right)$, tips for optimizing ventilator synchrony, $\mathrm{F}_{\mathrm{IO}_{2}}$ calculations with the use of the low-flow oxygen system, advantages to using a blender rather than bleeding oxygen into the system, salient points to consider when transitioning a patient from an ICU ventilator with tubing compensation to the LTV 1200, and inspiratory time limitations of volume control ventilation on the LTV 1200. Time was allotted for discussion and demonstration of each key point that was reviewed.

\section{Data Collection and Analytical Plan}

The pre- and post-assessment scores for each participant were entered into a data spreadsheet for analysis. Descriptive statistics reported participant demographics. Paired $t$ tests compared pre- and post- assessment scores for each participant. Statistical significance was established at $P<.05$.

\section{Results}

Figure 2 illustrates the impact the rapid cycle improvements (ie, ventilator mounts for the mobile ICUs, new batteries and carrying packs) had on addressing the equipment and process barriers to portable ventilator use with pediatric patients.

A majority of boot camp participants $(82 \%)$ were female. Most of the participants $(41 \%)$ had between 6 and 9 years of longevity in the field and 5 years of experience with our transport team. Demographic data are found in Table 1. Of the domains assessed, completing the ventilator pre-use check and engaging alarms showed the least improvement after the interactive educational intervention (Table 2). Most participants had difficulty selecting the mode of ventilation (pressure control rather than volume control) for infants requiring ventilation with a small $\mathrm{V}_{\mathrm{T}}$ and adjusting parameters to optimize patient-ventilator syn- 
Table 1. Demographics of the Ventilator Bootcamp Participants

\begin{tabular}{|c|c|c|c|c|c|c|c|c|c|}
\hline \multicolumn{2}{|c|}{ Age Groups } & \multicolumn{2}{|c|}{ Gender } & \multicolumn{2}{|c|}{$\begin{array}{l}\text { Post-Secondary } \\
\text { Education }\end{array}$} & \multicolumn{4}{|c|}{ Longevity } \\
\hline $\begin{array}{c}\text { Age } \\
\text { Range, y }\end{array}$ & $n(\%)$ & $\begin{array}{l}\text { Male, } \\
n(\%)\end{array}$ & $\begin{array}{c}\text { Female, } \\
n(\%)\end{array}$ & $\begin{array}{l}\text { Degree } \\
\text { Completed }\end{array}$ & $n(\%)$ & $\begin{array}{c}\text { Experience in } \\
\text { Respiratory Care, y }\end{array}$ & $n(\%)$ & $\begin{array}{l}\text { Air/Ground Transport } \\
\text { Experience, y }\end{array}$ & $n(\%)$ \\
\hline $18-23$ & $0(0)$ & $3(17)$ & $14(83)$ & Associate & $8(47)$ & $<5$ & $0(0)$ & $<5$ & $6(35)$ \\
\hline $24-32$ & $6(35)$ & & & Bachelors & $9(53)$ & $6-9$ & $7(41)$ & $6-9$ & $4(24)$ \\
\hline $33-40$ & $5(29)$ & & & & & $10-14$ & $4(24)$ & $10-14$ & $3(17)$ \\
\hline $41-49$ & $6(35)$ & & & & & $15-19$ & $5(29)$ & $15-19$ & $3(17)$ \\
\hline $50-59$ & $0(0)$ & & & & & $>20$ & $1(5)$ & $>20$ & $1(5)$ \\
\hline $60+$ & $0(0)$ & & & & & & & & \\
\hline
\end{tabular}

Table 2. Average Scores for the Major Domains Assessed for Each of the Scenarios

\begin{tabular}{|c|c|c|c|c|c|c|c|c|}
\hline & \multicolumn{2}{|c|}{$\begin{array}{l}\text { Perfoms all pre } \\
\text { use procedures }\end{array}$} & \multicolumn{2}{|c|}{$\begin{array}{c}\text { Selects initial } \\
\text { ventilator } \\
\text { settings (including } \\
\text { mode } \\
\text { of ventilation) }\end{array}$} & \multicolumn{2}{|c|}{$\begin{array}{l}\text { Engages alarms } \\
\text { according to policy }\end{array}$} & \multicolumn{2}{|c|}{$\begin{array}{l}\text { Completes all } \\
\text { safety checks }\end{array}$} \\
\hline & Pre & Post & Pre & Post & Pre & Post & Pre & Post \\
\hline Scenario 1 & $53 \%$ & $65 \%$ & $55 \%$ & $71 \%$ & $12 \%$ & $35 \%$ & $59 \%$ & $80 \%$ \\
\hline Scenario 2 & $71 \%$ & $71 \%$ & $69 \%$ & $74 \%$ & $33 \%$ & $14 \%$ & $75 \%$ & $78 \%$ \\
\hline Scenario 3 & $86 \%$ & $79 \%$ & $28 \%$ & $84 \%$ & $81 \%$ & $48 \%$ & $60 \%$ & $82 \%$ \\
\hline
\end{tabular}

Table 3. Comparison of Test Scores Before and After Educational Intervention

\begin{tabular}{|c|c|c|c|c|c|c|}
\hline & \multicolumn{2}{|c|}{ Scenario 1} & \multicolumn{2}{|c|}{ Scenario 2} & \multicolumn{2}{|c|}{ Scenario 3} \\
\hline & Pre & Post & Pre & Post & Pre & Post \\
\hline Mean, \% & 54 & 69 & 55 & 49 & 60 & 68 \\
\hline Min, $\%$ & 7 & 13 & 30 & 13 & 30 & 43 \\
\hline Max, \% & 87 & 100 & 93 & 77 & 97 & 100 \\
\hline SD & 0.2 & 0.2 & 0.2 & 0.2 & 0.2 & 0.2 \\
\hline$P$ of $t$ test & \multicolumn{2}{|c|}{.006} & \multicolumn{2}{|c|}{.10} & \multicolumn{2}{|c|}{.07 } \\
\hline
\end{tabular}

Data are reported as a $\%$ correct. $P$ values are from the $t$ test results comparing raw scores before and after the educational intervention.

chrony by accessing the sub-menu. Overall, most scores improved after the educational intervention. Table 3 provides a comparison of the test scores before and after the educational intervention. The impact of the ventilator boot camp on portable ventilator use during interfacility transport is shown in Figure 2.

\section{Discussion}

Rapid-cycle process improvements or PDSA cycles combine a continuous process of small tests of change with a longitudinal measurement process. ${ }^{5}$ This process offered our staff the ability to participate in solutions to problems they encountered in the field and to lead interventions to produce a positive impact. Although the PDSA cycles addressing process, equipment securing, and battery life issues did improve ventilator use on transport, it did not fully address all of the barriers that would have enabled our program to reach the benchmark for portable ventilator use during transport. The electronic health record review was helpful in understanding the types of patients with whom the RTs used manual ventilation rather than the LTV 1200 during transport. Through the retrospective chart review, we were able to identify the situations in which crew members had the most difficulty with portable ventilator use. This information was used to formulate the assessment and educational interventions that formed the ventilator boot camp.

The pre-assessment phase of the boot camp provided some insight as to why the crew identified an equipment issue on the fishbone diagram, such as "the ventilator doesn't work with small patients." Most RTs (69\%) did not select pressure control ventilation as the mode of ventilation that would provide an adequate inspiratory time for the patient. For example, for scenario involving the 2-month-old infant, a $\mathrm{V}_{\mathrm{T}}$ of $50 \mathrm{~mL}$ would be appropriate, but when volume control ventilation is selected the $50-\mathrm{mL}$ $\mathrm{V}_{\mathrm{T}}$ only allows for a 0.3 -second inspiratory time. Pressure 
control ventilation is the most appropriate mode because it allows for a more physiologically normal inspiratory time. Staff had difficulty assessing the sub menu. The sub-menu provided ventilator parameter options to optimize trigger and cycle synchrony. The pre-assessment facilitated not only the skills assessment, but enabled our team to have a better understanding of why the RT reported that the ventilator doesn't work with small children, this perception that was rooted the patient's inability to tolerate the mode of ventilation because of patient-ventilator asynchrony. Therefore, a portion of the educational intervention focused on mode selection, patient assessment, and methods to optimize patient-ventilator synchrony. This allowed the educators to interact with the RTs and guide them to the mode and settings that would address the respiratory insufficiency or failure and provide safe, effective ventilation.

The chart review also verified that the staff had limited exposure to NIV use for pediatric patients during interfacility transport. During the pre-assessment phase, only $28 \%$ of participants selected the initial inspiratory and expiratory pressure settings during invasive pressure control ventilation and NIV that would produce a $\mathrm{V}_{\mathrm{T}}$ in the lungprotective strategy range and adjusted the settings in response to the simulated patient's response to therapy, which was reflected in end-tidal $\mathrm{CO}_{2}$ and $\mathrm{S}_{\mathrm{pO}_{2}}$ changes. A minority of participants (28\%) accessed the sub-menu to optimize trigger and cycle sensitivity.

The respiratory therapists participating in the boot camp had both professional longevity and experience in the transport environment. However, the pre-assessment found that there was a need to address knowledge gaps that prevented appropriate use of a portable ventilator which had the potential to impair the RT's ability to distinguish between equipment failure (the ventilator is not working properly) and patient intolerance (patient-ventilator asynchrony). Our neonatal/pediatric critical care transport team completes $>3,000$ air and ground missions per year. Given that, in 2015 , only 172 pediatric patients $(6 \%)$ required ventilatory assistance after stabilization at the referral facility and transport to the pediatric ICU, ventilatory use for this population was a high-risk, low-volume procedure. This limited exposure to the LTV 1200 ventilator during transport could have contributed to knowledge gaps. The literature reports limited ventilator exposure may lead to mismanagement of ventilated patients, which could result in increased morbidity and mortality. ${ }^{6}$

Educational interventions have been used in health care to improve knowledge and critical-thinking skills. Workshops, web-based education, and simulation-based training have demonstrated positive outcomes. However, the literature reports that the most effective intervention is a combination of both didactic and simulated trainings. ${ }^{7}$ The intervention portion of the boot camp, which was an interactive educational session, provided the RT with the information needed to ad- dress the knowledge gaps identified in the pre-assessment phase. This portion of the boot camp provided RT with the ability to practice what they learned in a simulated environment with the ventilator. The staff was guided through structured improvement plans that allowed each RT the opportunity to direct the learning session to address his or her perceived knowledge gaps and learning needs.

The ventilator boot camp we conducted yielded results similar to those reported by Yee et $\mathrm{al}^{8}$ in their work with resident physicians. Similar to what is reported in the literature, the ventilator boot camp provided a safe environment in which simulation-based education could be used to achieve clinical skill goals and fill the gap when traditional clinical experience may be lacking. ${ }^{9}, 10$ Post-intervention assessment revealed an improvement in overall knowledge. The overall impact of the program can be seen in the sustained improvement in portable ventilator use during transport.

This process allowed our team to evaluate our previous training methods. Although the LTV 1200 was included in competency training prior to the boot camp, the "train the trainer" technique was used as a teaching and assessment method. This non-standardized approach could have contributed to problems such as missed teaching points or inconsistent approaches to assessing or addressing errors and knowledge gaps. The boot camp allowed our educators to standardize and evaluate our teaching and competency assessment process. The participants expressed that more frequent training sessions would be the most helpful aid in their day-to-day use of the ventilator.

There were several limitations to our study. The sample size was small, and our study was conducted at a single institution. The results of the pre-intervention assessment were not shared with staff. Lack of information regarding their pre-assessment score may have minimized the importance with which the staff perceived the educational intervention. If the RT had been aware of a knowledge gap, he or she may have viewed participation in the simulated hands-on portion of the educational intervention as more important. Another limitation is that the boot camp was limited to RT. Our 3-person transport team is composed of a RT, a nurse and a paramedic. Although the nurses and paramedics participated in the PDSA cycles identifying the process and equipment issues, they were not included in the ventilator boot camp. Including the entire team in the boot camp may improve team function and recognition of patient intolerance or equipment malfunction during transport of mechanically ventilated children.

\section{Conclusions}

Comparative quality-performance data were useful in identifying the need for knowledge and competency assessment. The pre-assessment provided evidence of knowl- 


\section{Ventilator Boot CAmp for Pediatric Interfacility Transport}

edge gaps. Post-assessment results validated the need to conduct education and competency assessment at defined intervals, perhaps more frequently than on an annual basis. Assessments on a more frequent basis for high-risk, lowvolume use of pressure control and NIV may have facilitated sustained improvement in quality performance.

\section{REFERENCES}

1. Almada Bassani M, Mezzacappa Filho F, de CC, Tadeu MM. An evaluation of peak inspiratory pressure, tidal volume, and ventilatory frequency during ventilation with a neonatal self-inflating bag resuscitator. Respir Care 2012;57(4):525-530.

2. Lee HM, Cho KH, Choi YH, Yoon SY, Choi YH. Can you deliver accurate tidal volume by manual resuscitator? Emerg Med J 2008; 25(10):632-634.

3. Bigham MT, Schwartz HP, Ohio Neonatal/Pediatric Transport Quality Collaborative. Quality metrics in neonatal and pediatric critical care transport: a consensus statement. Pediatr Crit Care Med 2013; 14(5):518-524.

4. Amiri H, Gholipour C, Mokhtarpour M, Shams Vahdati S, Hashemi Aghdam Y, Bakhshayeshi M. Two-day primary trauma care work- shop: early and late evaluation of knowledge and practice. Eur J Emerg Med 2013;20(2):130-132.

5. Crowl A, Sharma A, Sorge L, Sorensen T. Accelerating quality improvement within your organization: applying the model for improvement. J Am Pharm Assoc 2015;55(4):e364-e374.

6. Slutsky AS. History of mechanical ventilation. From Vesalius to ventilator-induced lung injury. Am J Respir Crit Care Med 2015; 191(10):1106-1115.

7. Cook DA, Hatala R, Brydges R, Zendejas B, Szostek JH, Wang AT, et al. Technology-enhanced simulation for health professions education: a systematic review and meta-analysis. JAMA 2011;306(9): 978-988.

8. Yee J, Fuenning C, George R, Hejal R, Haines N, Dunn D, et al. Mechanical ventilation boot camp: a simulation-based pilot study. Crit Care Res Pract 2016;2016:4670672.

9. Wayne DB, Cohen ER, Singer BD, Moazed F, Barsuk JH, Lyons EA, et al. Progress toward improving medical school graduates' skills via a "boot camp" curriculum. Simul Healthc 2014;9(1): 33-39.

10. McGaghie WC, Issenberg SB, Cohen ER, Barsuk JH, Wayne DB. Does simulation-based medical education with deliberate practice yield better results than traditional clinical education? A meta-analytic comparative review of the evidence. Acad Med 2011;86(6): 706-711. 